\title{
Epidermal differentiation does not involve the pro-apoptotic executioner caspases, but is associated with caspase-14 induction and processing
}

\author{
S Lippens ${ }^{1}$, M Kockx², M Knaapen ${ }^{2}$, L Mortier ${ }^{3}$, \\ R Polakowska ${ }^{3}$, A Verheyen ${ }^{2}$, M Garmyn $^{4}$, A Zwijsen $^{5}$, \\ P Formstecher $^{3}$, D Huylebroeck ${ }^{5}$, P Vandenabeele $^{\star, 1,6}$ and \\ W Declercq ${ }^{1,6}$ \\ ${ }^{1}$ Molecular Signaling and Cell Death Unit, Department of Molecular Biology, \\ Flanders Interuniversity Institute for Biotechnology and Ghent University, \\ K.L. Ledeganckstraat 35, B-9000 Ghent, Belgium \\ 2 Department of Pathology, APCAM and Dermatology, University Hospital \\ Middelheim, Lindendreef 1, B-2020 Antwerpen, Belgium \\ 3 Inserm U459, Faculté de Medicine Henri Warembourg, Place de Verdun 1 , \\ 59045 Lille, France \\ ${ }^{4}$ Department of Dermatology, Catholic University of Leuven, Herestraat 49, \\ B-3000 Leuven, Belgium \\ ${ }^{5}$ Department of Cell Growth, Differentiation and Development, Laboratory of \\ Molecular Biology, Flanders Interuniversity Institute for Biotechnology and \\ Catholic University of Leuven, Herestraat 49, B-3000 Leuven, Belgium. \\ ${ }^{6}$ Both authors share senior authorship \\ * Corresponding author: Peter Vandenabeele, Molecular Signaling and Cell \\ Death Unit, Department of Molecular Biology, Flanders Interuniversity Institute \\ for Biotechnology and Ghent University, K.L. Ledeganckstraat 35, B-9000 \\ Ghent, Belgium. Tel: ++32 (0)9 264 8716; Fax: ++32 (0)9 264 5348; \\ E-mail: peter.vandenabeele@dmb.rug.ac.be
}

Received 22.9.00; accepted 27.9.00

Edited by G Melino

\begin{abstract}
The epidermis is a stratified squamous epithelium in which keratinocytes progressively undergo terminal differentiation towards the skin surface leading to programmed cell death. In this respect we studied the role of caspases. Here, we show that caspase-14 synthesis in the skin is restricted to differentiating keratinocytes and that caspase-14 processing is associated with terminal epidermal differentiation. The proapoptotic executioner caspases $-3,-6$, and -7 are not activated during epidermal differentiation. Caspase-14 does not participate in apoptotic pathways elicited by treatment of differentiated keratinocytes with various death-inducing stimuli, in contrast to caspase-3. In addition, we show that non-cornifying oral keratinocyte epithelium does not express caspase- 14 and that the parakeratotic regions of psoriatic skin lesions contain very low levels of caspase-14 as compared to normal stratum corneum. These observations strongly suggest that caspase-14 is involved in the keratinocyte terminal differentiation program leading to normal skin cornification, while the executioner caspases are not implicated. Cell Death and Differentiation (2000) 7, 1218-1224.
\end{abstract}

Keywords: caspase; skin; keratinocyte; differentiation; psoriasis
Abbreviations: $\mathrm{CHX}$, cycloheximide; NHK, normal human keratinocytes; SE, skin equivalent

\section{Introduction}

Apoptosis is achieved by an evolutionarily conserved pathway in which cysteinyl aspartate-specific proteinases, known as caspases, play a central role. ${ }^{1}$ Caspases are synthesized as zymogens, comprising a prodomain, as well as a large (p20) and small (p10) subunit. They become activated by proteolytic cleavage at Asp residues between the different subdomains, which leads to formation of mature caspase, a tetramer consisting of two p20/p10 heterodimers. There are two main activation pathways for procaspases during apoptosis. In the extrinsic pathway the initiator procaspase8 becomes activated by proximity-induced autoactivation due to recruitment by the adaptor protein FADD (Fas-associated death domain) to the death domain-containing receptors of the TNF receptor superfamily, such as TNF-R1 (tumor necrosis factor-receptor 1) and Fas. ${ }^{2,3}$ The intrinsic death pathway, triggered for instance by cytotoxic drugs or DNA damage, is controlled by mitochondrial release of cytochrome c. Cytochrome $c$ together with dATP binds the adaptor Apaf-1 (apoptosis activating factor-1), which leads to recruitment and activation of procaspase-9. ${ }^{4,5}$ The two pathways converge on the proteolytic activation of the apoptosis-related, shortprodomain effector caspases-3, -6 and -7 .

The epidermis is a stratified squamous epithelium in which keratinocytes are organized in distinct cell layers. During embryogenesis the single-layered embryonic ectoderm becomes a bilayered epithelium as a layer of peridermal cells is formed on its surface. Upon stratification of the epidermis and formation of its definitive layers of the epidermis, the periderm is gradually degraded. ${ }^{6}$ In adult skin the basal cells are proliferation competent, but suprabasal keratinocytes do not proliferate and undergo programmed cell death in the final stage of differentiation, viz. the formation of the cornified layers. The molecular mechanism of this process is not well understood. There are few reports on a relationship between caspases and terminal keratinocyte differentiation. Recently it was demonstrated that caspase-1, -2, -3, -4 and -7 mRNA is expressed in cultured keratinocytes. ${ }^{7}$ Making use of in vivo and in vitro reconstituted epidermis, others suggested that procaspase-3 is activated during late keratinocyte differentiation. ${ }^{8}$

We previously identified mouse and human caspase-14. ${ }^{9}$ This caspase has a restricted expression pattern opposed to all other caspases identified, ${ }^{9-11}$ since caspase-14 mRNA could not be detected in neither heart, lung, muscle, kidney, spleen, thymus, brain nor testis. In contrast, caspase-14 mRNA was expressed in the embryo 
and the skin. ${ }^{9,12}$ Here, we document that the expression of caspase-14 protein in the epidermis is restricted to differentiated keratinocytes. Processed caspase-14 was found in skin whereas the pro-apoptotic caspases such as caspase-3 and -7 were only present as precursor forms. Caspase-14 does not participate in apoptotic pathways, but its processing is associated with epidermal differentiation. In addition, we show that in the parakeratotic regions of psoriatic skin lesions the caspase-14 levels are very low as compared to normal stratum corneum.

\section{Results}

\section{Caspase-14 synthesis in the skin is restricted to differentiating keratinocytes}

The tissue distribution of caspase-14 mRNA was shown to be restricted to the embryo and the adult skin. ${ }^{9,12}$ To further investigate where caspase-14 is expressed in the skin, we performed in situ mRNA hybridization and immunohistochemistry on embryonic and adult skin sections. In situ localization of mouse caspase-14 mRNA in the skin of the 15.5 d.p.c. embryo revealed that caspase-14 mRNA is exclusively expressed in the differentiated, suprabasal layers of the epidermis (Figure 1A). Using a polyclonal anti-mousecaspase-14 antiserum, recognizing both mouse and human caspase-14, we confirmed that caspase-14 protein synthesis is restricted to differentiating keratinocytes in embryonic skin (Figure 1B). Caspase-14 was found both in the cytoplasm and the nucleus. It has been reported previously that in addition to their cytoplasmic localization, caspases are also present in other subcellular fractions and organelles. ${ }^{13-15}$ Caspase-14 is not expressed in the embryonic periderm and the basal keratinocyte layer, which represents undifferentiated keratinocytes and do not contain the typical differentiation markers of adult skin. ${ }^{16}$ Immunohistochemistry on adult mouse and human skin sections showed that the caspase-14 protein is expressed from the spinous layers to the stratum granulosum and the stratum corneum (Figure 1C,D). Keratinocytes from the stratum spinosum and the stratum granulosum both exhibit cytoplasmic and nuclear caspase-14 staining. As differentiation proceeds, the nucleus becomes degraded and the cornified layers are formed. The hair shaft and the sebaceous gland consists of differentiated keratinocytes, ${ }^{17}$ and also here caspase-14 was present (Figure 1E,F). In the eccrine sweat glands, which are located in the dermis but are of epidermal origin, caspase-14 was not expressed (Figure $1 \mathrm{G})$. Moreover, caspase-14 expression was also absent in the oral epithelium (Figure $1 \mathrm{H}$ ). This epithelium shows a multilayered architecture similar to that of the skin, with the exception of cornification. Taken together these data strongly suggest a function for caspase-14 in skin differentiation.

\section{Caspase processing in normal epidermis in vivo}

To analyze whether caspase-14 and other caspases are present in an activated state in normal skin, we tested lysates of mouse skin by immunoblotting with different polyclonal anticaspase antisera. In the case of caspase-14 we detected both the proenzyme and the p20 fragment, indicative for processed
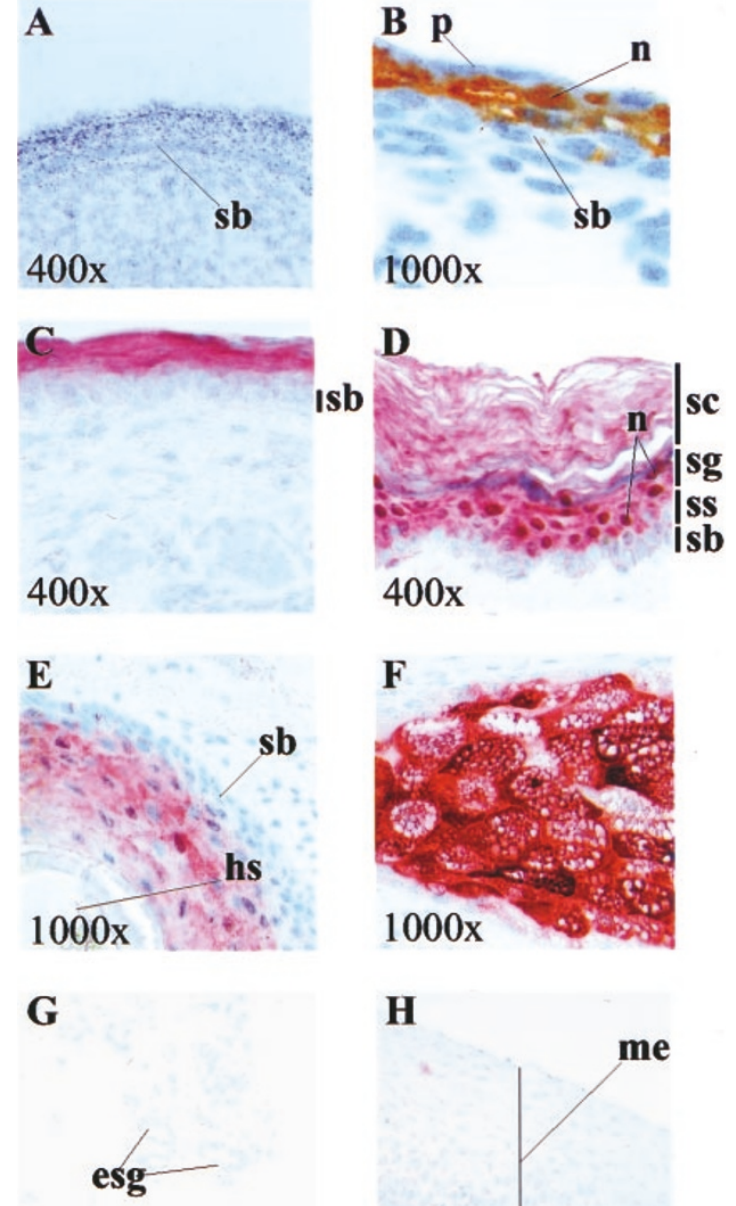

200x

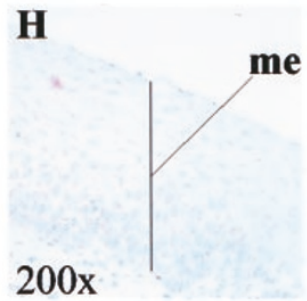

Figure 1 Caspase-14 is expressed in the suprabasal layers of the skin 15.5 d.p.c. BALB/c embryo tissue (A, B), tail of adult BALB/c mice (C), adult human skin tissue $(\mathbf{D}, \mathbf{E}, \mathbf{F}, \mathbf{G})$ and human oral epithelium $(\mathbf{H})$ were fixed, paraffin embedded and sliced. In situ mRNA hybridization (A) and immunohistochemistry $(\mathbf{B}-\mathbf{H})$ revealed that caspase-14 is expressed in the suprabasal layers of the epidermis, both in cytoplasm and nucleus $(\mathbf{A}, \mathbf{B}, \mathbf{C}$, D), but is absent in the periderm (B). Caspase-14 is expressed in epidermal derivatives such as hair follicle $(\mathbf{E})$ and sebaceous gland $(\mathbf{F})$, while it is absent in the eccrine sweat glands $(\mathbf{G})$ or the oral epithelium $(\mathbf{H})$. esg, eccrine sweat gland; hs, hair shaft; me, multi-layered oral epithelium; $n$, nucleus; $p$, periderm; $\mathrm{sb}$, stratum basale; sc, stratum corneum; sg, stratum granulosum; ss, stratum spinosum

caspase-14 (Figure 2). The 20 and $18.5 \mathrm{kDa}$ polypeptides likely represent the large subunit (p20) with and without prodomain, respectively. The typical apoptosis-related caspases, such as caspase-3, -6 and -7 , were only present as unprocessed proenzymes. Consequently, we stained sections of human skin with different anti-caspase-3 antibodies recognizing total caspase-3 or detecting only activated caspase-3 (Figure 3). Staining with the antibody against total caspase-3 revealed that caspase-3 was expressed throughout the whole epidermis (Figure $3 \mathrm{~A}$ ). When stained with the activation-specific anti-caspase-3 antibody we consistently observed no staining in the epidermal layers of the skin (Figure 3B). In parallel we also stained with the anti-caspase- 
3 antibodies on a tumor section containing apoptotic cells. All tumor cells stained with the antibody against total caspase- 3 antibodies on a tumor section containing apoptotic cells. All tumor cells stained with the antibody against total caspase-3 but only the apoptotic cells within the tumor did stain for active caspase-3, showing that the antibody functioned as expected (Figure 3C). In addition, we were not able to detect processed caspase-3 in human skin lysates by Western blotting (data not shown). So our data do not support a general role for caspase-3 in the terminal keratinocyte differentiation program.

\section{Expression and processing of caspase-14 in human keratinocytes is inducible in vitro}

Since caspase-14 is not present in the stratum basale but is expressed in the differentiating layers of the epidermis, we analyzed whether the protein expression of caspase-14

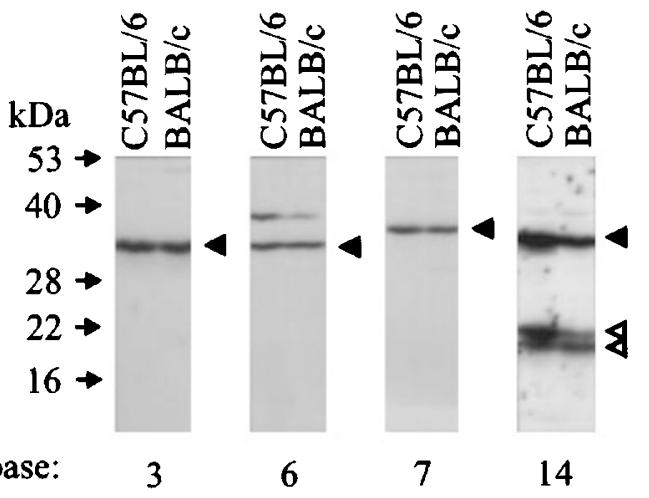

Figure 2 Caspase protein expression profile in normal mouse skin. $100 \mu \mathrm{g}$ of protein extracted from skin of $\mathrm{BALB} / \mathrm{C}$ and $\mathrm{C} 57 \mathrm{BL} / 6$ mice was used for immunoblotting to analyze the activation of caspases (filled arrowheads, procaspase; open arrowheads, cleaved caspase fragments representing the large p20 subunit) in keratinocytes could be induced in vitro by conditions of differentiation. In a Western blot experiment we found that undifferentiated NHK or HaCaT cells did not express caspase-14 (Figure 4A). Primary human keratinocytes grown under growth-arresting conditions (e.g. suspension or confluency) express several differentiation markers, such as keratin-1 and -10 , involucrin and transglutaminase. ${ }^{18}$ In both $\mathrm{NHK}$ and $\mathrm{HaCaT}$ cells grown postconfluently, caspase-14 expression was induced together with involucrin (Figure 4A). This is not a general characteristic of caspases, as procaspase-3 levels were not affected when cells were grown postconfluently (data not shown). This differentiation condition did not lead to processing of
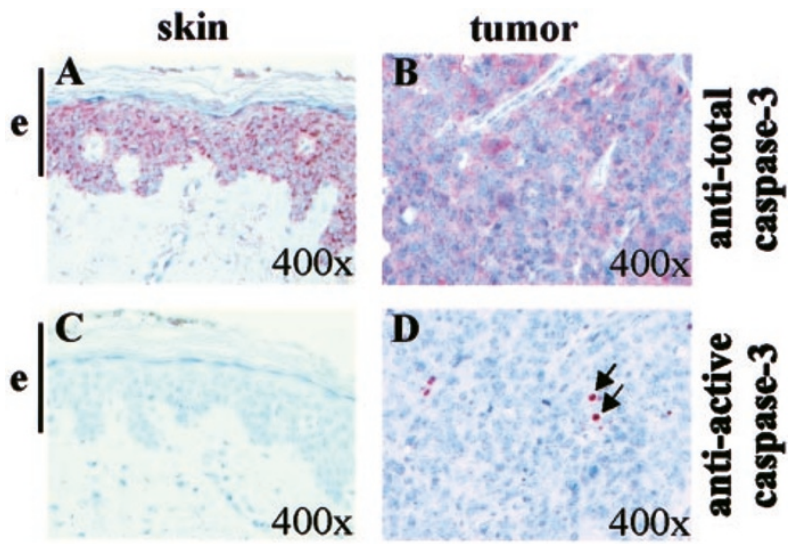

Figure 3 Caspase-3 is not activated in normal human epidermis. Caspase-3 is not activated in normal human epidermis. Human skin $(\mathbf{A}, \mathbf{C})$ and tumor tissue (B, D) were fixed, embedded in paraffin and sliced. The sections were used for immunohistochemical staining with an antibody recognizing total caspase-3 (A, B) or with an antibody specific for activated caspase-3 (C, D). Caspase- 3 was expressed in epidermis, dermis and tumor. Epidermis (e) is negative for staining with the activation-specific anti-caspase-3 antibody. Arrows show apoptotic tumor cells containing activated caspase- 3
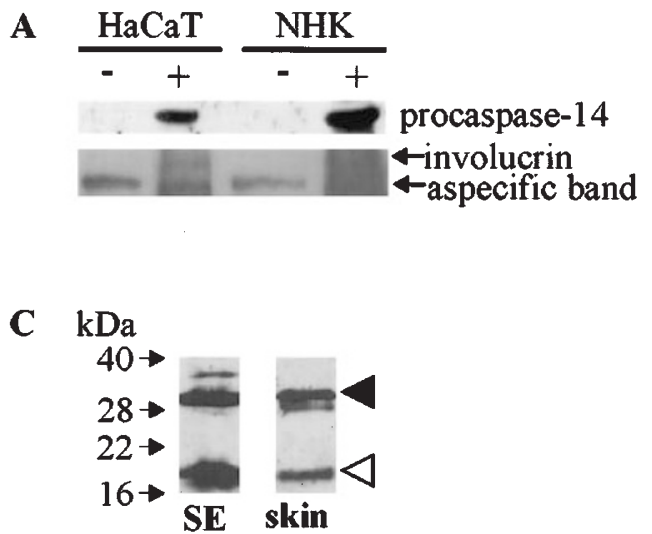

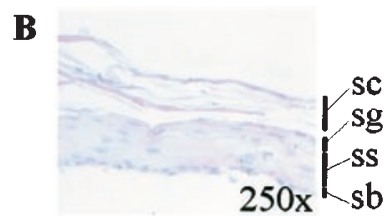

D

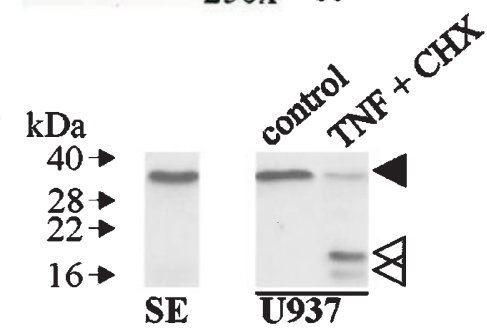

Figure 4 Caspase-14 expression is induced and processed during in vitro keratinocyte differentiation. (A) Lysates of undifferentiated ( -$)$ and differentiated (+) $\mathrm{HaCaT}$ and NHK cells were used for immunoblotting. Differentiation was induced by growing cells postconfluency. Western blots were analyzed for caspase-14 or involucrin expression. (B) Sections of human skin equivalents were used for eosine/haematoxyline staining. The different epidermal layers were formed, including the stratum corneum, indicating that the keratinocytes underwent the complete program of terminal differentiation. Lysates of human skin equivalents were analyzed by means of immunoblotting for processing of caspase-14 (C) and caspase-3 (D) (filled arrowheads, procaspase; open arrowheads, cleaved caspase fragments representing the large p20 subunit). Lysates derived from adult human skin and TNF/CHX treated U937 cells were used as controls for caspase-14 processing and caspase-3 processing respectively. sb, stratum basale; sc, stratum corneum; SE, skin equivalent; sg, stratum granulosum; ss, stratum spinosum 
caspase-3 or -14 , as only the precursor forms could be revealed.

To analyze caspase-14 expression and processing in a more physiological relevant keratinocyte differentiation assay we used an in vitro epidermal equivalent system. ${ }^{19}$ As shown in Figure 4B, the in vitro skin equivalent developed a normal stratum corneum. Under these conditions both procaspase-14 and a p20 subunit fragment, indicative for caspase-14 activation, were demonstrated (Figure 4C). Caspase-3 was not processed since only its proform could be detected (Figure 4D). These data are in agreement with our observation that also in vivo caspase-14 synthesis and processing is correlated with epidermal differentiation (Figure 1).

\section{Caspase-14 is not involved in the apoptotic pathway}

Caspase-14 contains a putative short prodomain and hence the procaspase is structurally related to the apoptotic executioner caspases, such as caspase-3, -6 and -7 , which require upstream initiator caspases to become activated. ${ }^{1}$ In vitro, caspase-14 can be processed by the initiator caspases8 and $-10 .^{9,20}$ We evaluated whether caspase -14 can be part of an apoptotic caspase cascade in keratinocytes. Growtharrested NHK cells, but not HaCaT cells, become resistant to apoptosis, ${ }^{21}$ therefore HaCaT cells were differentiated to express caspase-14 and were subsequently treated with $\mathrm{TNF} / \mathrm{CHX}$. As shown in Figure 5, confluency of HaCaT cells induced caspase-14 synthesis. Upon combined addition of TNF and $\mathrm{CHX}$ both differentiated and undifferentiated cells died in an apoptotic way characterized by clear morphological features, such as membrane blebbing and nuclear fragmentation (Figure 5A). Lysates were made and analyzed for caspase- 3 and -14 processing in a Western blotting experiment (Figure 5B,C). As expected, the combination of TNF and $\mathrm{CHX}$ resulted in a clear reduction in level of intact procaspase-3. In addition, processed fragments corresponding to the caspase-3 p20 subunit (with and without short prodomain) became evident after $\mathrm{TNF} / \mathrm{CHX}$ treatment. Activation of caspase-3 correlated with detection of cleaved PARP, a prototype caspase-3 substrate. ${ }^{22}$ In contrast, caspase-14 processing was not observed, indicating that this caspase does not become activated during keratinocyte apoptosis. Similar results were obtained after treatment of the keratinocytes with other apoptotic stimuli such as UVB irradiation or staurosporine (data not shown). Together, these data demonstrate that caspase-14 is an atypical short-prodomain caspase that does not participate in the apoptotic cascade, in contrast to the other short-prodomain caspases such as caspase- $3,-6$ and -7 .

\section{Synthesis of caspase-14 is disturbed in parakeratotic regions of psoriatic lesions}

To evaluate a potential role for caspase-14 in skin pathologies, we analyzed biopsies from psoriatic skin plaques by immunohistochemistry with anti-caspase-14
$\mathbf{A}$

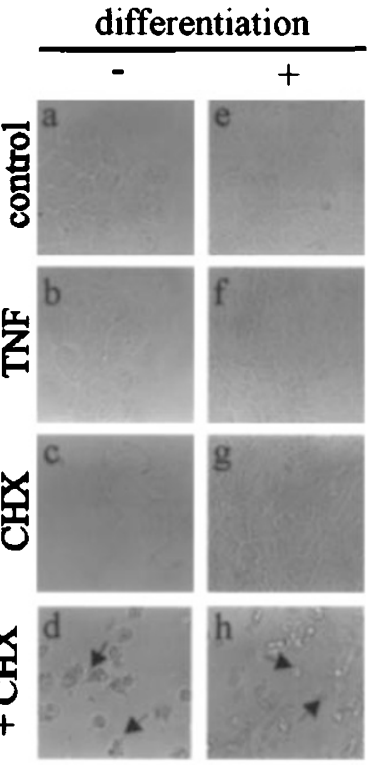

B

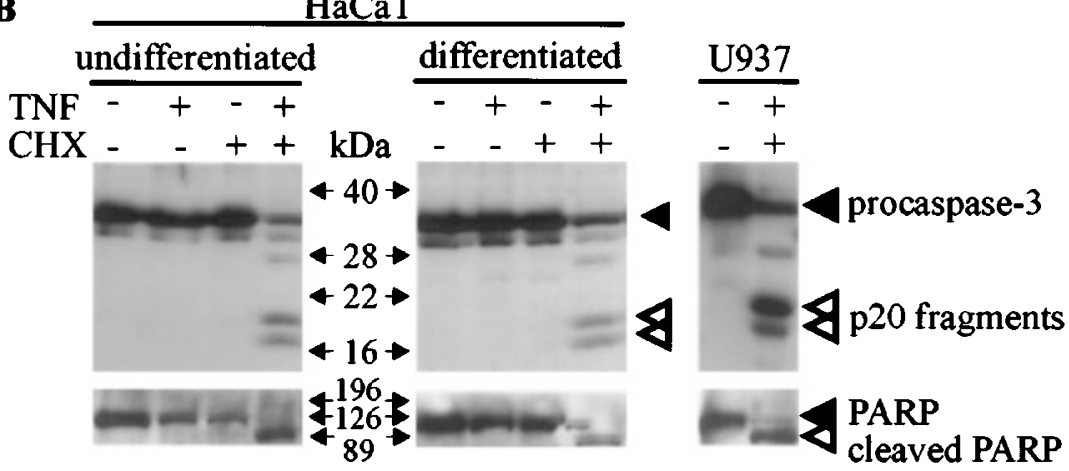

C

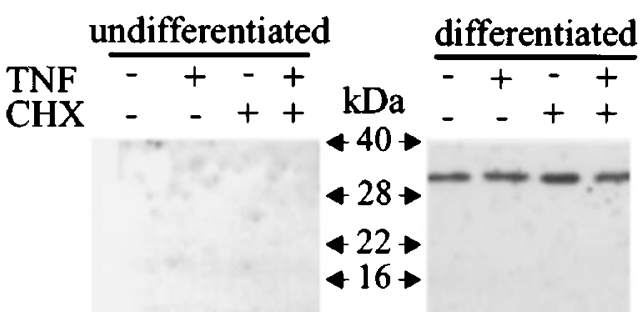

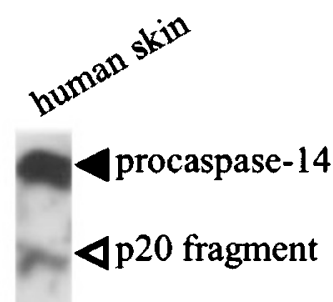

Figure 5 Caspase-14 is not processed in apoptotic conditions. HaCaT cells were grown in normal conditions or induced to differentiate by postconfluent growth. Cells were then treated with TNF $(1000 \mathrm{U} / \mathrm{ml})+\mathrm{CHX}(10 \mu \mathrm{g} / \mathrm{ml})$ to induce apoptosis. Pictures were taken after $12 \mathrm{~h}(\mathbf{A})$; arrows in $\mathrm{d}$ and $\mathrm{h}$ show apoptotic cells Lysates were analyzed by immunoblotting for processing of caspase-3 and cleaved PARP (B) as well as caspase-14 (C). Lysates derived from TNF/CHX treated U937 cells were used as control for caspase-3 processing, lysates from human epidermis were applied as control for caspase-14 processing. Filled arrowheads, precursor form; open arrowheads, processed form 
antiserum. Plaques from six different psoriatic patients were analyzed and compared to non-involved skin. Psoriatic skin showed suprabasal proliferation and disturbed keratinocyte differentiation (Figure 6), as reported earlier. ${ }^{23}$ Since psoriatic skin does not develop a normal stratum granulosum, in which keratinocyte enucleation takes place, these parakeratotic regions do retain their nuclei, resulting in an abnormal stratum corneum. Non-involved skin of psoriatic patients expressed caspase-14 in a pattern as discussed above (Figure 6A). In psoriatic lesions the caspase-14 expression pattern in the spinous layers was comparable to non-involved skin, indicating that the initial onset of caspase-14 expression is normal. Remarkably, the disturbed keratinization process in the parakeratotic region of psoriatic skin is associated with a very low level of caspase-14 synthesis (Figure 6B). Cytoplasmic caspase-14 staining was strongly reduced, as compared to the expression levels in the spineous layers, while nuclear caspase-14 was absent. This suggests that a deregulation of caspase-14 synthesis may be related to disturbance of the normal, terminal keratinocyte differentiation program in psoriatic patients.

\section{Discussion}

Unlike the apoptosis- and inflammation-related caspases, caspase-14 has a very restricted expression pattern. ${ }^{9,12}$ Here we extended our previous study and examined caspase-14 expression in skin. In situ mRNA hybridization in embryonic skin revealed that caspase-14 mRNA is expressed in the differentiating suprabasal keratinocytes, and not in the basal layer; this was confirmed by immunohistochemistry. The periderm was negative for caspase-14 protein, although the periderm has desmosomes and can express the differentiation marker involucrin. ${ }^{24}$ In adult mouse and human skin, all suprabasal layers stain positive for caspase-14 protein. It is, however, possible that activation of caspase-14 is confined to more specific layers. In embryonic and adult skin, caspase-14 protein is located in both cytoplasm and nucleus. Originally it
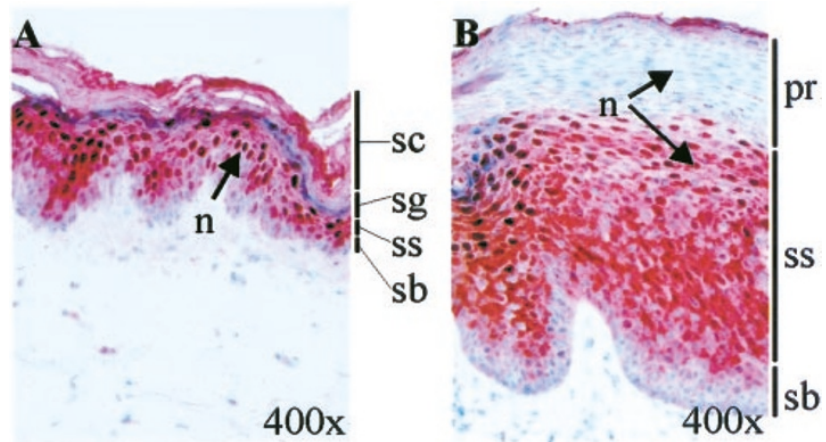

Figure 6 Caspase-14 synthesis is downregulated in parakeratotic regions of psoriatic lesions. Sections of normal skin $(\mathbf{A})$ and psoriatic plaque tissue (B) derived from the same patient were prepared and used for caspase-14 detection by immunostaining. In normal skin caspase-14 was present in al suprabasal layers. In psoriasis-involved skin the caspase-14 expression level in the spineous layers is comparable to normal epidermis, while in the parakeratotic region caspase-14 expression is clearly downregulated. $n$, nucleus, pr, parakeratotic region, sb, stratum basale, sc, stratum corneum, sg, stratum granulosum, ss, stratum spinosum was assumed that caspases are exclusively cytosolic, but recently it has become clear that they can reside in different cellular compartments, including the nucleus. The large prodomains of caspase-1 and -2 have been shown to be involved in their nuclear localization. ${ }^{14,25}$ Although the mechanism used by short-prodomain caspases for nuclear translocation is not clear, nuclear accumulation of caspase-14 may be part of the keratinocyte differentiation program. Skin derivatives, such as hair follicles and the sebaceous gland, that consist of differentiated keratinocytes, also express caspase-14. The eccrine sweat gland comprises a coiled tube connected to the surface by a duct. Unlike the epidermis and the hair follicle, the sweat glands do not regularly renew themselves and they express an atypical set of cytokeratins. ${ }^{26,27}$ Hence, since sweat glands do not express caspase-14, it is tempting to speculate that caspase-14 fulfils a role in epidermal keratinocyte differentiation. Caspase-14 expression is not related to stratification as such, because another stratifying keratinocyte epithelium, viz. mouth epithelium, does not show any detectable caspase-14 staining. In contrast to skin the mouth epithelium is a noncornifying mucosal epithelium, suggesting that caspase-14 synthesis may be correlated with the process of cornification.

We analyzed the activation state of caspase-14 and other caspases in normal skin and showed that processed caspase-14 fragments of the expected size could be detected in skin lysates. The apoptosis-related effector caspases-3, -6, and -7 were only present as proforms. We could not demonstrate the presence of activated caspase-3 neither in skin nor in in vitro skin equivalents. Processing of caspase-3 in skin differentiation has been reported previously, ${ }^{8}$ although the reported fragments did not correspond to the typical $19-17 \mathrm{kDa}$ or $12 \mathrm{kDa}$ bands on Western blot. Obviously, one cannot exclude the possibility that the apparent conflicting data are due to a different source of skin samples. Our data argue against a major involvement of the apoptosis-related executioner caspases in normal skin differentiation. In fact, it has been suggested that $\mathrm{NF}-\kappa \mathrm{B}$ activation in the suprabasal layers prevents premature apoptosis to allow terminal keratinocyte differentiation. $^{28}$

It has previously been shown that in vitro translated procaspase-14 can be processed, though rather weakly, by treatment with purified caspase-8 and -10 , and not with other caspases, ${ }^{9,20}$ suggesting that caspase-14 might operate in an apoptotic proteolytic cascade. Surprisingly, neither extrinsic nor intrinsic cell death stimuli resulted in caspase-14 processing. However, human caspase-14 displays a putative VGGD caspase cleavage site between its p20 and p10 subunit. Ahmad et al. ${ }^{20}$ also showed that caspase-14 is not cleaved or activated, unlike caspase-3 and -7 , by addition of cytochrome $c$ and dATP to $\mathrm{S} 100$ cellular extracts. These data strongly indicate that the physiological trigger for caspase-14 processing is not an apoptotic caspase cascade and that caspase-14 can be considered as a atypical short-prodomain effector caspase, unrelated to apoptosis. It is not necessarily a caspase that should cleave and hence activate caspase-14, since serine proteases have been reported to be able to activate procaspases. $^{29,30}$ 
When keratinocyte cultures were differentiated by applying a growth-arresting treatment, such as postconfluency, procaspase-14 synthesis is strongly induced but not processed. However, this differentiation model does not reflect normal epidermal differentiation and these cultures possess little or no anucleate corneocytes. ${ }^{31}$ When NHK cells were used to generate in vitro skin equivalents processing of caspase-14 could be induced to a comparable extent as observed in normal skin lysates. To date there are no indications which are the targets of activated caspase-14, since no substrates have been described. Taken together, these results suggest that caspase-14 processing is probably not an early event in keratinocyte differentiation but is associated with the formation of normal cornified epidermal architecture.

Psoriasis is characterized by keratinocyte hyperproliferation, loss of granular layer and parakeratotic differentiation. ${ }^{32,33}$ So far, this epidermal hyperproliferation has not been explained on a molecular basis and it is still unclear whether the major defect resides within the keratinocyte, the immune compartment or both. ${ }^{34}$ Since there is loss of granular layer in psoriasis, the nucleus is not degraded, which gives rise to an abnormal parakeratotic stratum corneum. In these parakeratotic regions caspase-14 expression is very low in the cytoplasma and absent in the nucleus. This observation adds to the relationship between caspase-14 synthesis and processing and normal epidermal keratinocyte cornification, viz. degradation of the nucleus. Strategies to upregulate caspase-14 expression may constitute a valuable therapeutic contribution to diseases involving parakeratosis, including psoriasis.

\section{Materials and methods}

\section{Keratinocyte culture and differentiation}

The immortalized keratinocyte HaCaT cell line, ${ }^{35}$ was obtained from Dr N Fusenig (German Cancer Research Center, Heidelberg, Germany) and the cells were grown in DMEM supplemented with $10 \%$ FCS. Normal human keratinocytes (NHK) were isolated from foreskins of young donors as described. ${ }^{36}$ NHK cells were grown in keratinocyte serum-free medium (Life Technologies, Paisley, UK) with a calcium concentration of $0.09 \mathrm{mM}$ and were supplemented with $50 \mu \mathrm{g} / \mathrm{ml}$ of bovine pituitary extract and $5 \mathrm{ng} / \mathrm{ml}$ of human epidermal growth factor (Life Technologies, Paisley, UK). Third to fifth passage cells were used. HaCaT or NHK cells were grown post confluently in the same medium as used for keratinocyte culture. Skin equivalents (SE) were prepared from the first or second passage of human epidermal keratinocytes grown in KGM medium (Clonetics) and fibroblasts grown in RPMI1640 plus 10\% FCS as described earlier. ${ }^{19}$ Mature SE were paraffin embedded and used for haematoxylin/eosin staining to evaluate their differentiation status or lysed for Western blot analysis.

\section{Apoptosis induction in keratinocyte cultures}

HaCaT cells were induced to differentiate by postconfluency. Apoptosis was induced by treatment with a combination of $1000 \mathrm{IU} /$ ml human TNF and $10 \mu \mathrm{g} / \mathrm{ml}$ cycloheximide (CHX), $35 \mathrm{~mJ} / \mathrm{cm}^{2}$ UVB or $100 \mathrm{ng} / \mathrm{ml}$ staurosporine. As a control, cells were untreated or were treated with $1000 \mathrm{IU} / \mathrm{ml}$ TNF or $10 \mu \mathrm{g} / \mathrm{ml} \mathrm{CHX}$. Twelve hours after treatment, lysates were prepared using caspase lysis buffer (1\% NP40, $200 \mathrm{mM} \mathrm{NaCl}, 10 \mathrm{mM}$ Tris $\mathrm{HCl}$ pH 7.5, $5 \mathrm{mM}$ EDTA, 10\% glycerol, $1 \mathrm{mM}$ PMSF, $0.3 \mathrm{mM}$ aprotinin, $1 \mathrm{mM}$ leupeptin and $1 \mathrm{mM}$ oxidized glutathione). Purified E. coli-derived TNF was prepared in our laboratory and had a specific biological activity of $0.94 \times 10^{8} \mathrm{IU} / \mathrm{mg}$ as compared to international standards for TNF quantification derived from the National Institute for Biological Standards and Control (Potters Bar, UK).

\section{Immunoblot analysis}

Transfected cells, differentiated keratinocytes or skin tissue were lysed in caspase lysis buffer, protein concentration was determined and equal protein amounts were submitted to polyacrylamide gel electroforesis and immunoblotted. Anti-involucrin and anti-human caspase-3 were purchased from Sigma Chemical Co. (St. Louis, MO, USA) and BioSource International (Camarillo, CA, USA), respectively. An antibody against poly(ADP-ribose) polymerase (PARP) was obtained from Biomol Research Laboratories (Plymouth Meeting, PA, USA). Antibodies against murine caspases were raised by injecting rabbits with purified, bacterially expressed caspase preparations. ${ }^{37}$ Note that anti-murine caspase- 14 antibody crossreacts with human caspase-14 both on Western blots and in immunohistochemical staining. The polyclonal rabbit anti-mouse caspase antibodies recognize both procaspases and activated caspases in Western blotting experiments (data not shown).

\section{In situ mRNA hybridization and immunohistochemistry}

Mouse embryos, aged 15.5 days post coitum (d.p.c.), as well as skin tissues from humans and adult C57BL/6 mice were fixed in $4 \%$ paraformaldehyde in phosphate buffered saline (PBS), after which they were embedded in paraffin and sliced $(4-6 \mu \mathrm{m})$. Endogenous peroxidase was blocked with peroxidase blocking reagent (Dako, Carpinteria, CA, USA). A rabbit polyclonal antibody originally raised against recombinant, bacterially expressed mouse caspase- 3 or -14 stained mouse and human homologs. Active caspase- 3 was detected in tissue sections using activation statespecific polyclonal anti-caspase-3 antibody (PharMingen, San Diego, CA, USA). The primary antibody was detected by an indirect peroxidase antibody conjugate technique. The sections were incubated with a rat anti-rabbit antibody (Dako, Carpinteria, CA, USA) for $45 \mathrm{~min}$, and 3-amino-9-ethylcarbazole (Dako, Carpinteria, CA, USA) was used as chromogen. Radioactive in situ hybridization on paraffin sections was performed as described. ${ }^{38} \mathrm{~A}{ }^{35} \mathrm{~S}$-labeled antisense mouse caspase-14 RNA probe was used.

\section{Acknowledgments}

The authors wish to thank $E$ van Damme and $M$ De Bie for technical assistance, $W$ Drijvers and $M$ Vandecasteele for editorial help, and $A$ Raeymaekers for recombinant cytokine purification. $P$ Vandenabeele is a Research Associate and M Kockx is a post-doctoral Research Assistant with the FWO-Vlaanderen. Research was supported by grants of the Interuniversitaire Attractiepolen (contract nr. P4/26), and EC-RTD program (QLRT-199-00739) and a grant of the Fonds voor Wetenschappelijk Onderzoek-Vlaanderen (No. 9005097N). 


\section{References}

1. Earnshaw WC, Martins LM and Kaufmann SH (1999) Mammalian caspases: structure, activation, substrates and functions during apoptosis. Ann. Rev. Biochemistry 68: 383-424

2. Wallach D, Boldin M, Varfolomeev E, Beyaert R, Vandenabeele P and Fiers W (1997) Cell death induction by receptors of the TNF family: towards a molecular understanding. FEBS Lett. 410: 96-106

3. Schulze-Osthoff K, Ferrari D, Los M, Wesselborg S and Peter ME (1998) Apoptosis signaling by death receptors. Eur. J. Biochem. 254: 439-459

4. Li P, Nijhawan D, Budihardjo I, Srinivasula SM, Ahmad M, Alnemri ES and Wang X (1997) Cytochrome $c$ and dATP-dependent formation of Apaf-1/caspase- 9 complex initiates an apoptotic protease cascade. Cell 91: 479-489

5. Pan G, O'Rourke K and Dixit VM (1998) Caspase-9, Bcl-XL, and Apaf-1 form a ternary complex. J. Biol. Chem. 273: 5841-5845

6. Holbrook KA (1983) Structure and function of the developing human skin. In Biochemistry and physiology of the skin, Vol. 1, Goldsmith, ed (New York: Oxford University Press) pp. 64-101

7. Takahashi T, Ogo M and Hibino T (1998) Partial purification and characterization of two distinct types of caspases from human epidermis. J. Invest. Dermatol. 111 $367-372$

8. Weil M, Raff MC and Braga VM (1999) Caspase activation in the terminal differentiation of human epidermal keratinocytes. Curr. Biol. 9: 361-364

9. Van de Craen M, Van Loo G, Pype S, Van Criekinge W, Van den brande I, Molemans F, Fiers W, Declercq W and Vandenabeele P (1998) Identification of a new caspase homologue: caspase-14. Cell Death Differ. 5: 838-846

10. Van de Craen M, Vandenabeele P, Declercq W, Van den brande I, Van Loo G, Molemans F, Schotte P, Van Criekinge W, Beyaert R and Fiers W (1997) Characterization of seven murine caspase family members. FEBS Lett. 403 61-69

11. Van de Craen M, Van Loo G, Declercq W, Schotte P, Van den brande I, Mandruzzato S, van der Bruggen P, Fiers W and Vandenabelle P (1998) Molecular cloning and identification of murine caspase-8. J. Mol. Biol. 284: 1017- 1026

12. Hu S, Snipas SJ, Vincenz C, Salvesen G and Dixit VM (1998) Caspase-14 is a novel developmentally regulated protease. J. Biol. Chem. 273: 29648-29653

13. Martins LM, Mesner PW, Kottke PW, Basi GS, Sinha GS, Tung JS, Svingen PA Madden BJ, Takahashi A, McCormick DJ, Earnshaw WC and Kaufmann SH (1997) Comparison of caspase activation and subcellular localization in HL-60 and K562 cells undergoing etoposide-induced apoptosis. Blood 90: 4283-4296

14. Colussi PA, Harvey NL and Kumar S (1998) Prodomain-dependent nuclear localization of the caspase-2 (Nedd2) precursor. J. Biol. Chem. 273: 2453524542

15. Zhivotovsky B, Samali A, Gahm A and Orrenius S (1999) Caspases: their intracellular localization and translocation during apoptosis. Cell Death Differ. 6 644-651

16. Byrne C, Tainsky $M$ and Fuchs E (1994) Programming gene expression in developing epidermis. Development 120: 2369-2383

17. Hardy MH (1992) The secret life of the hair follicle. Trends Genet. 8: 55-61

18. Poumay $Y$ and Pittelkow MR (1995) Cell density and culture factors regulate keratinocyte commitment to differentiation and expression of suprabasal K1/K10 keratins. J. Invest. Dermatol. 104: 271-276

19. Haake A and Polakowska RR (1995) UV-induced apoptosis in skin equivalents: Inhibition by phorbol ester and Bcl-2 overexpression. Cell Death Differ. 2: $183-$ 193

20. Ahmad M, Srinivasula SM, Hegde R, Mukattash R, Fernandes-Alnemri T and Alnemri ES (1998) Identification and characterization of murine caspase-14, a new member of the caspase family. Cancer Res. 15: 5201-5205
21. Chaturvedi V, Qin J-Z, Denning MF, Choubey D, Diaz MO and NickoloffBJ (1999) Apoptosis in proliferating, senescent, and immortalized keratinocytes. J. Biol. Chem. 274: $23358-23367$

22. Lazebnik YA, Kaufmann SH, Desnoyers S, Poirier GG and Earnshaw WC (1994) Cleavage of poly(ADP-ribose) polymerase by a proteinase with properties like ICE. Nature 371: $346-347$

23. Henseler T (1997) The genetics of psoriasis. J. Am. Acad. Dermatol. 37: S1 - 11

24. WattFM, Keeble S, Fisher C, Hudson DL, Codd J and Salisbury JR (1989) Onset of expression of peanut lectin-binding glycoproteins is correlated with stratification of keratinocytes during human epidermal development in vivo and in vitro. J. Cell. Sci. 94: 355-359

25. Mao PL, Jiang Y, Wee BY and Porter AG (1998) Activation of caspase-1 in the nucleus requires nuclear translocation of procaspase- 1 mediated by its prodomain. J. Biol. Chem. 273: 23621-23624

26. Sato K (1993) Biology of the eccrine sweat gland. In Dermatology in General Medicine, Fitzpatrick TB, Eisen AZ, Wolff K, Freedberg IM and Austen KF, eds (New York: McGraw-Hill) pp. 221-241

27. Schön M, Benwood J, O'Connell-Willstaed T and Rheinwald JG (1999) Human sweat gland myoepithelial cells express a unique set of cytokeratins and reveal the potential for alternative epithelial and mesenchymal differentiation states in culture. J. Cell Sci. 112: 1925-1936

28. Seitz CS, Freiberg RA, Hinata $K$ and Khavari PA (2000) NF- $\kappa B$ determines localization and features of cell death in epidermis. The J. Clin. Invest. 105:253260

29. Zhou $Q$ and Salvesen GS (1997) Activation of pro-caspase-7 by serine proteases includes a non-canonical specificity. Biochem. J. 324: 361-364

30. Schotte P, Van Criekinge W, Van de Craen M, Van Loo G, DesmedtM, Grooten J, Cornelissen M, De Ridder L, Vandekerckhove J, Fiers W, Vandenabeele P and Beyaert R (1998) Cathepsin B-mediated activation of the proinflammatory caspase-11. Biochem. Biophys. Res. Commun. 251:379-387

31. Green $H$ (1977) Terminal differentiation of cultured human epidermal cells. Cell 11: $405-416$

32. Ragaz $A$ and Ackerman $A B$ (1979) Evolution, maturation, and regression of lesions of psoriasis. New observations and correlation of clinical and histologic findings. Am. J. Dermatopathol. 1: 199-214

33. Bata-Csorgo Z, Hammerberg C, Voorhees JJ and Cooper KD (1993) Flow cytometric identification of proliferative subpopulations within normal human epidermis and the localization of the primary hyperproliferative population in psoriasis. J. Exp. Med. 178: 1271-1281

34. Bos JD and De Rie MA (1999) The pathogenesis of psoriasis: immunological facts and speculations. Immunol. Today 20: 40-46

35. Boukamp P, Petrussevska RT, Breitkreuz D, Hornung J, Markham A and Fusenig NE (1988) Normal keratinization in a spontaneously immortalized aneuploid human keratinocyte cell line. J. Cell Biol. 106: 761-771

36. Kitano Y and Okada N (1983) Separation of the epidermal sheet by dispase. Br. J. Dermatol. 108: 555-560

37. Van de Craen M, Declercq W, Van den brande I, Fiers W and Vandenabeele P (1999) The proteolytic procaspase activation network: an in vitro analysis. Cell Death Differ. 6: 1117-1124

38. Dewulf N, Verschueren K, Lonnoy O, Moren A, Grimsby S, Vande Spiegle K, Miyazono K, Huylebroeck D and ten Dijke P (1995) Distinct spatial and temporal expression patterns of two type I receptors for bone morphogenetic proteins during mouse embryogenesis. Endocrinology 136: 2652-2663 